\title{
Dynamique du cholestérol et des acides biliaires. Aspects comparatifs
}

\author{
C Lutton \\ Université de Paris XI, Bât 447, Laboratoire de physiologie de la nutrition, URA 646 CNRS, \\ 91405 Orsay Cedex
}

(Reçu le 16 novembre 1989; accepté le 21 décembre 1989)

\begin{abstract}
Résumé - Si la concentration de cholestérol d'un tissu donné reste similaire chez le rat, le porc ou l'homme, l'importance relative des processus régissant les entrées (absorption et synthèse) et les sorties (excrétions fécales de cholestérol et d'acides biliaires) du système cholestérol diffère beaucoup d'une espèce à l'autre. Le rat, dont la cholestérolémie s'élève peu après addition de cholestérol à l'alimentation (animal "hyporépondeur"), adapte bien sa biosynthèse des acides biliaires aux variations de l'entrée du cholestérol dans l'organisme. Ce processus rend compte de 80 à $85 \%$ des sorties de cholestérol, l'excrétion fécale de cholestérol étant un processus mineur; ce dernier est la conséquence d'une pauvre sécrétion de cholestérol hépatique dans la bile due à la faible hydrophobicité de ses principaux acides biliaires. De plus, cet animal présente une synthèse intestinale importante de cholestérol et d'apolipoprotéines $\left(B_{48}\right.$, en particulier...) sécrétés sous la forme de lipoprotéines très légères (chylomicrons et VLDL) à renouvellement plasmatique plus rapide que les VLDL (à apo $B_{100}$, E...) sécrétées par le foie. Les "remnants" des VLDL du rat sont essentiellement captés très rapidement par le foie, leur voie de transformation intraplasmatique en IDL et LDL étant peu importante $(\leq 10 \%)$. L'homme, qui présente une hypercholestérolémie plus marquée après l'ingestion de cholestérol exogène (sujet ehyperrépondeur"), semble posséder un pouvoir de transformation du cholestérol en acides biliaires moins modulable. Ce processus rend compte de $50 \%$ seulement des sorties de cholestérol, l'excrétion fécale de cholestérol étant quantitativement aussi importante. La concentration de cholestérol et le rapport cholestérol/acides biliaires sont beaucoup plus élevés dans la bile humaine que dans la bile murine, les acides biliaires principaux y étant plus hydrophobes. Si l'intestin et le foie contribuent à la cholestérogenèse, l'importance relative de ce dernier organe est probablement plus grande chez l'homme que chez le rat. De même, une plus grande fraction des VLDL plasmatiques est transformée en IDL et LDL, ces dernières lipoprotéines représentant le transporteur principal du cholestérol plasmatique. Rechercher si les différences que nous venons de souligner dans les processus de la biodynamique du cholestérol du rat, d'une part, et de l'homme, d'autre part, sont généralisables aux Mammifères peu ou très sensibles à l'hypercholestérolémie et à l'athérosclérose, nous semble une voie de recherche fondamentale pour les prochaines années.
\end{abstract}

analyse compartimentale / biosynthèse du cholestérol et des acides biliaires / sócrétion blllaire de cholestérol / cholestérolémle / sécrétion des apolipoprotéines

Summary - Cholesterol and bile acid dynamics. Comparative aspects. While the cholesterol concentration in a given tissue is similar in the rat, pig or man, the relative importance of the processes regulating the input (absorption and synthesis) and output (faecal cholesterol and bile acid excretions) of the cholesterol system is very different from one species to another. The rat, whose cholesterolaemia does not significantly increase after cholesterol addition to the diet ("hyporesponding" animal), successfully adapts its bile acid biosynthesis to variations in cholesterol input. This process accounts for 80 to $85 \%$ of cholesterol output, faecal cholesterol excretion being a 
minor process. The latter results from a low liver cholesterol secretion in the bile due to the low hydrophobicity of its main bile acids. Furthermore, in this animal a high intestinal synthesis of cholesterol and apolipoproteins (particularly $B_{48}$ ) is observed. The latter are secreted as very light lipoproteins (chylomicrons and VLDL) with a faster plasma turnover than the VLDL (apoB ${ }_{100}$, E...) secreted by the liver. The "remnants" of rat VLDL are essentially very rapidly taken up by the liver; their interplasmatic transformation pathway into $I D L$ and $L D L$ is not very significant $(\leq 10 \%)$. Man, who has a more significant hypercholesterolaemia after exogenous cholesterol ingestion ("hyperresponding" subject) seems to have a less modulable capacity for transforming cholesterol into bile acids. This process accounts for only $50 \%$ of cholesterol output, faecal cholesterol excretion being quantitively just as significant. Cholesterol concentration and the cholesterol/bile acid ratio are much higher in human than in rat bile, the main bile acids being more hydrophobic. While both the intestine and liver contribute to cholesterogenesis, the relative importance of the latter is probably greater in man than in the rat. Moreover, a larger fraction of plasma VLDL is transformed into IDL and LDL, the latter representing the main plasma cholesterol carrier. Determining whether the differences between the biodynamics of cholesterol processes in the rat and in man can be generalised to mammals with low or high sensitivities to hypercholesterolaemia and atherosclerosis seems to be a fundamental research objective for the next few years.

compartmental analysis / cholesterol and bile acid biosynthesis / biliary cholesterol secretion / cholesterolaemia / apolipoprotein secretion

\section{INTRODUCTION}

Si la connaissance de la structure du système cholestérol a beaucoup progressé au cours des dix dernières années chez les principales espèces de Mammifères utilisées par le biologiste (souris, rat, hamster, lapin, porc, singe, homme), la compréhension des facteurs qui modulent les deux principaux processus de renouvellement du cholestérol (biosynthèse et transformation en acides biliaires) est encore incomplète.

\section{CONCENTRATION ET RÉPARTITION DU CHOLESTÉROL DANS LES ORGANES}

Si, pour une même espèce, la concentration de cholestérol varie beaucoup d'un organe à lautre, elle est constante quel que soit le Mammifère, pour un tissu donné, à quelques exceptions près (fig 1). Ces exceptions concernent essentiellement le foie, les surrénales et le plasma. Le cho- lestérol total rend compte d'environ $0,2 \%$ du poids corporel. Sa répartition relative dans les divers tissus reflète beaucoup celle de leur concentration pondérale dans l'organisme. Une fraction plus ou moins importante du cholestérol dans les organes est susceptible de s'échanger avec le cholestérol du plasma : il a été nommé espace cholestérol ou encore cholestérol mobile (Chevallier, 1956, 1967a). Cette fraction qui représente seulement $25-30 \%$ du cholestérol total de la peau ou du cerveau atteint $100 \%$ pour la plupart des organes dont le foie (Chevallier, 1967b). Ainsi, le cholestérol mobile représente-t-il $65-75 \%$ du cholestérol total de l'organisme. It est l'objet d'échanges plus ou moins rapides avec le cholestérol du plasma.

\section{STRUCTURE DU SYSTĖME CHOLESTÉROL}

La topologie du cholestérol mobile dans l'organisme du Mammifère est représentée comme un système mammillaire ouvert dont le plasma constitue le compartiment 


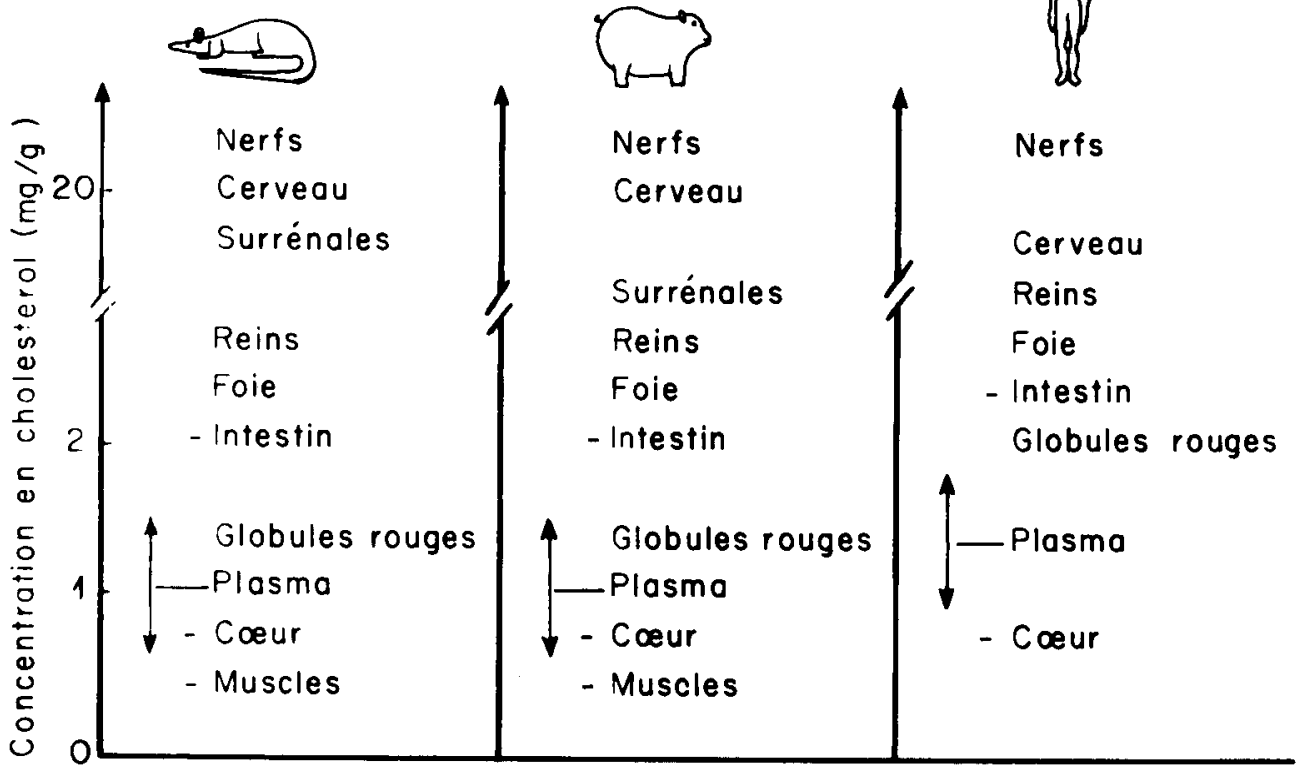

Fig 1. Concentration en cholestérol $(\mathrm{mg} / \mathrm{g})$ dans divers organes chez le rat, le porc et l'homme.

central et les organes les différents compartiments périphériques (fig 2). Certains d'entre eux jouent un rôle important. Le foie, où s'effectue une synthèse de cholestérol, est l'organe exclusif de la transformation du cholestérol en acides biliaires. L'intestin grêle, autre organe essentiel de la synthèse du cholestérol, représente le site spécifique de l'absorption du cholestérol alimentaire, biliaire ou provenant de la paroi même du tractus digestif.

Les relations extrinsèques du système cholestérol correspondent aux entrées (absorption et sécrétion interne) et aux sorties vers ou hors du système (excrétion fécale de cholestérol et biosynthèse des acides biliaires). Ces débits d'entrée ou de sortie (ou flux nets, mg/j) sont aisément mesurés par la méthode d'équilibre isotopique (Lutton et Chevallier, 1972) ou par celle du principe d'occupation (Bergner, 1964).
Les relations intrinsèques (exprimant les mouvements de cholestérol dans l'organisme, au sein du plasma ou entre le plasma et les organes) peuvent être appréhendées par la modélisation de systèmes élémentaires compartimentaux simples et, plus récemment, avec le développement de l'informatique, par la simulation de systèmes complexes multicompartimentaux (Champarnaud et al, 1987). La méthode du modèle, bien décrite par Magot (1989) consiste pour l'expérimentateur à caractériser son système, c'est-à-dire, dans un premier temps, à accumuler un certain nombre de connaissances acquises par des méthodes simples d'identification afin de définir la structure du modèle censé représenter le système étudié (fig 3). Dans un deuxième temps, il faut simuler le système caractérisé, c'est-à-dire comparer les données simulées et les données expéri- 


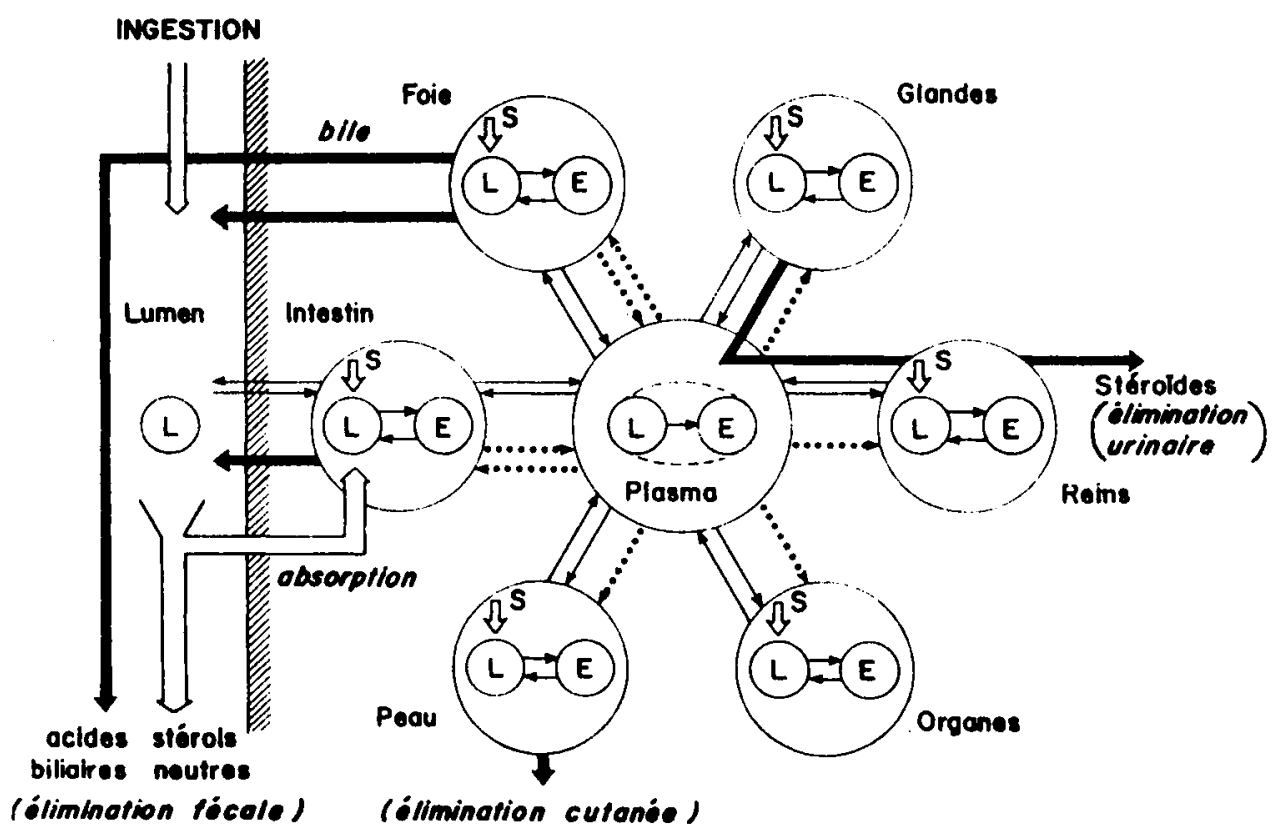

(D) cholestórol tlbre

(E) cholestórol estérifié

JS synthèse tlssulatro de cholestérol

coosonstert particulaire

- ellmination de métabolites du cholestérol

$\Longrightarrow$ change de cholestérol

$\longrightarrow$ transfert de cholestérol hors du syatème

Fig 2. Topologie du système cholestérol.

mentales. Cette comparaison définit l'écart, que l'on réduit au maximum en modifiant les valeurs des paramètres. Cette méthode aboutit donc au meilleur jeu de paramètres pour le modèle choisi ou, en cas d'échec, à proposer une nouvelle caractérisation. On observe donc que l'un des intérêts majeurs des études cinétiques et de la modélisation est de permettre une étude quantitative du métabolisme.

Devant la complexité du système cholestérol du rat, Magot a effectué une démarche en 5 étapes. Les 4 premières ont consisté en l'élaboration de modèles partiels par des marquages spécifiques, suivie de l'étude de l'évolution de la radioactivité dans les différentes parties. La dernière étape a concerné la synthèse des 4 modèles partiels en un modèle complet à 16 compartiments (fig 4). La validation du modèle a été assurée par la bonne adéquation entre les valeurs simulées et les valeurs expérimentales dans 10 conditions isotopiques différentes et par la bonne concordance avec les données de la littérature pour les paramètres et débits identi- 


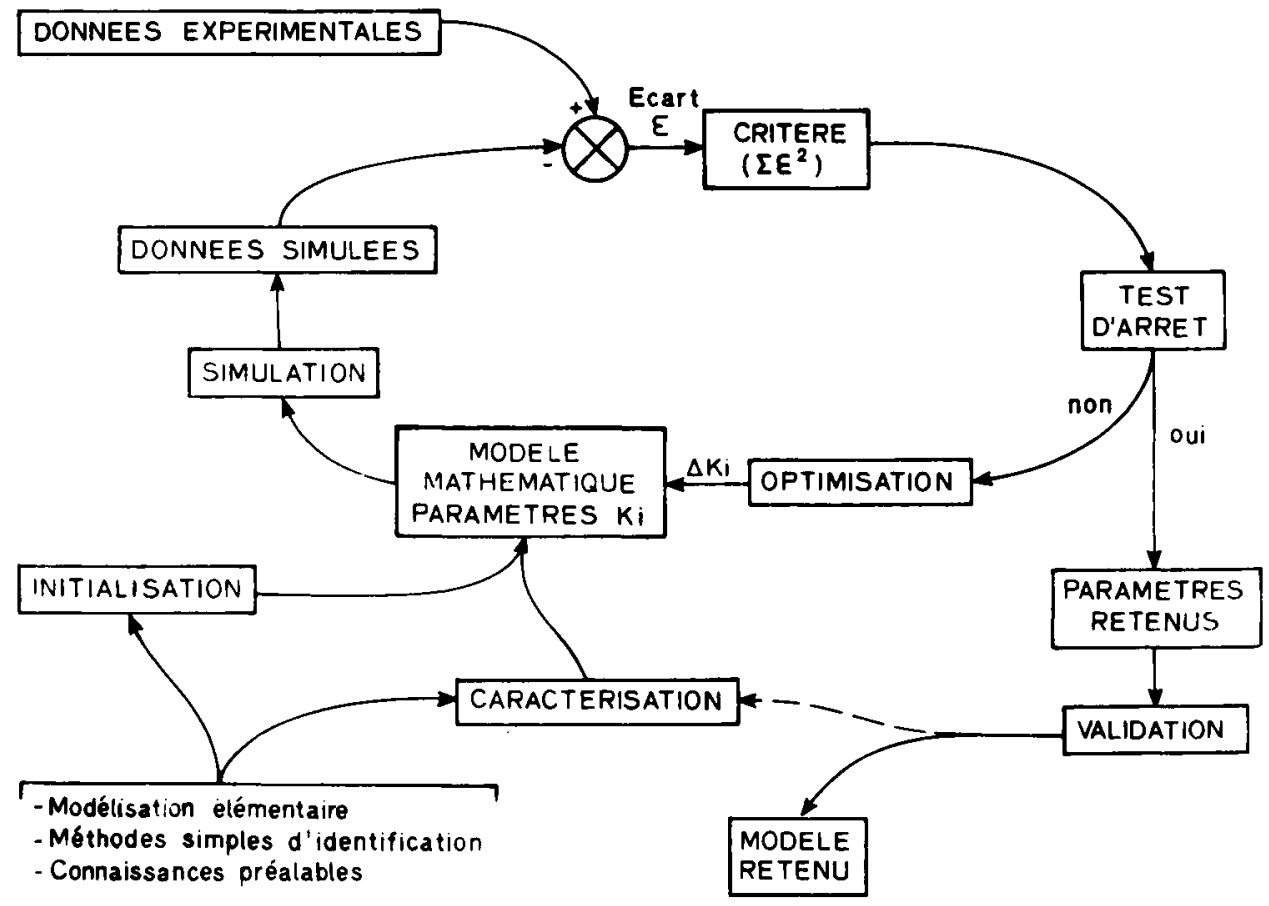

Fig 3. La méthode du modèle : procédure d'identification.

fiés. Au-delà de la simple représentation du système cholestérol, l'élaboration d'un tel modèle présente un intérêt à plusieurs niveaux. II permet de faire la synthèse des données connues sur la structure du système; il permet de déterminer in vivo des paramètres non mesurables par d'autres méthodes et de confirmer ou d'infirmer des hypothèses préalablement émises; enfin, c'est un outil à utiliser dans de nouvelles conditions nutritionnelles ou pathologiques du métabolisme du cholestérol. Donnons quelques exemples des données importantes acquises avec ce modèle chez le rat (Magot, 1989) :

- dans nos conditions (aliment semisynthétique donné une fois par jour), le rôle prépondérant de l'intestin dans le dé- versement du cholestérol de synthèse est à nouveau confirmé. Les VLDL intestinales sont plus particulièrement les pourvoyeurs du cholestérol de synthèse intestinale (Chevallier et Lutton, 1973; Lutton, 1976; Chevallier et Magot, 1975; Magot et Chevallier, 1979; Magot et al, 1985);

- les lipoprotéines légères (chylomicrons et VLDL) captent dans le plasma du cholestérol estérifié provenant des HDL (Magot, 1985);

- avec la captation hépatique des lipoprotéines légères, ce cholestérol aboutit au foie d'où il peut être éliminé sous forme d'acides biliaires ou de cholestérol. Par ce processus, les lipoprotéines légères intestinales jouent donc un rôle essentiel dans 
a
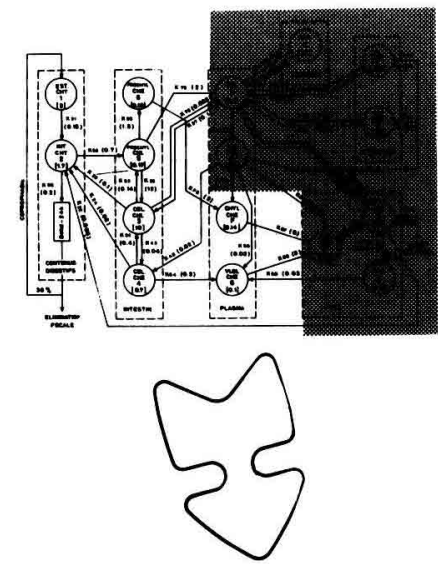

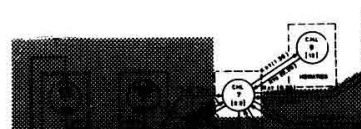

e

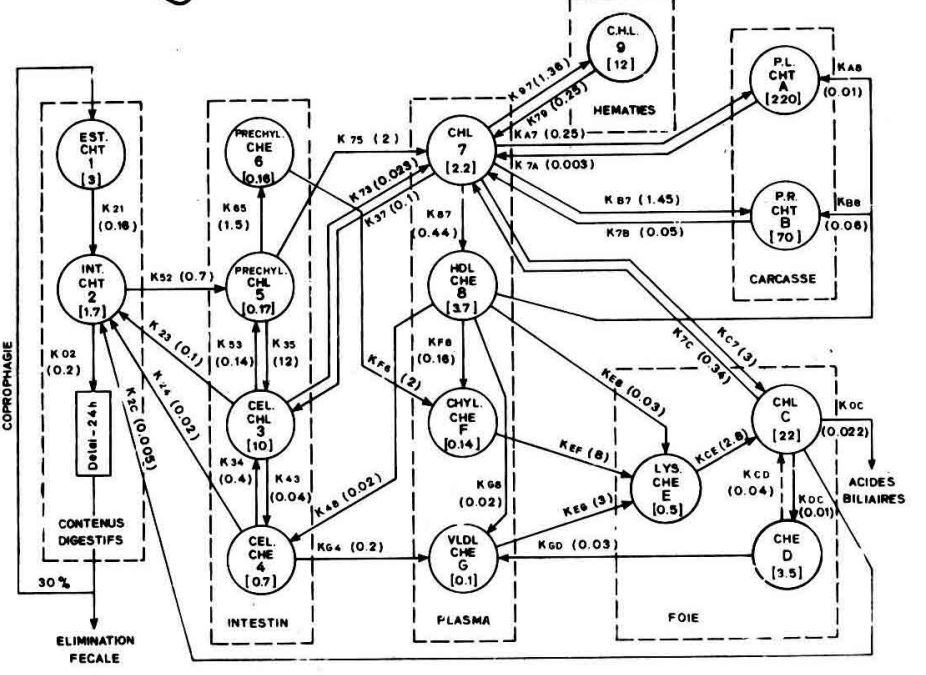

c
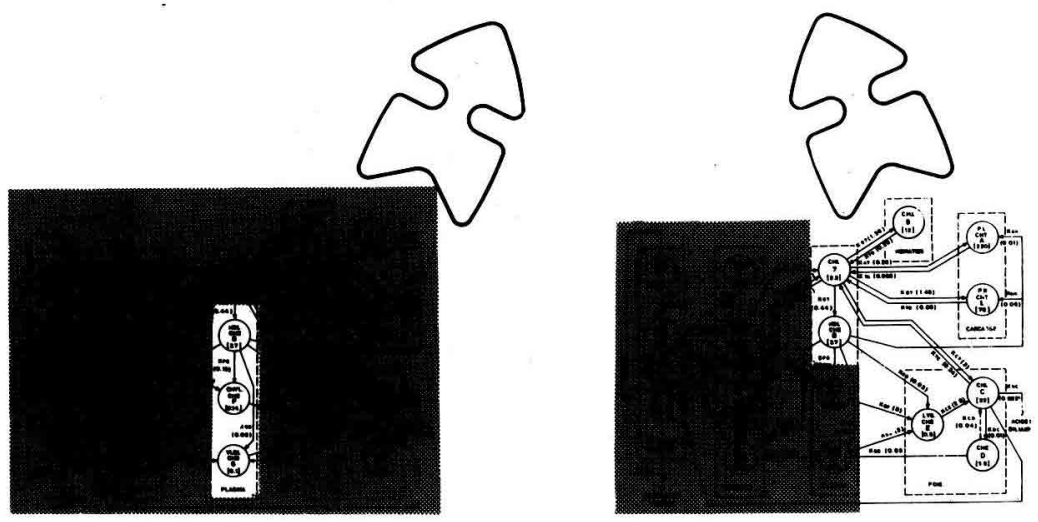

b 
l'épuration du cholestérol plasmatique et tissulaire (voir plus loin).

Deux autres points peuvent être soulignés et illustrés à partir de deux schémas simplifiés du modèle précédent. La figure 5 illustre les vitesses des flux bidirectionnels de cholestérol libre $\left(\mathrm{mg} \times \mathrm{h}^{-1}\right)$ entre le foie et le plasma, le plasma et les hématies, le plasma et le pool rapide. On remarque l'extrême rapidité des échanges de cholestérol libre plasma-foie; ces échanges bidirectionnels sont souvent considérés comme ne donnant pas lieu à un flux net de cholestérol : remarquons simplement qu'il est difficile de mettre en évidence expérimentalement une différence entre deux flux aussi rapides ! Le temps de renouvellement du cholestérol libre hépatique du rat est inférieur à $3 \mathrm{~h}$. II en est de même de celui du cholestérol libre hépatique de l'homme (Schwartz et al, 1982). On ne s'étonne donc plus d'observer que le cholestérol ou les acides biliaires de la bile aient une origine essentiellement plasmatique. Nous avons montré chez le rat, mais aussi chez l'homme (Férézou et al, 1987), que même dans des conditions pour lesquelles la synthèse du cholestérol est stimulée dans le foie (Férézou et al, 1989), il n'existe pas de déversement préférentiel de cholestérol de synthèse hépatique dans la bile. Ceci montre que le débit de cholestérol de synthèse hépatique est bien inférieur aux débits des échanges de cholestérol libre entre le plasma et le foie. Sa valeur correspond probablement à $2-5 \%$ de celle de ces derniers.

La figure 6 illustre d'une manière simplifiée les flux nets de cholestérol estérifié entre l'intestin, le plasma et le foie chez le rat. Rappelons que le modèle original n'a pu être développé qu'en tenant compte d'une hétérogénéité du cholestérol ester de l'intestin et du cholestérol estérifié dans le plasma, hétérogénéité probablement en liaison avec celle des apo $B$ (apo $B_{1}$ et apo $B_{h}$ ) dont les origines et les destinées métaboliques diffèrent (voir plus loin). Soulignons simplement que, hormis la vitesse de pénétration hépatique du cholestérol estérifié des chylomicrons vers le foie, qui est 6-10 fois inférieure aux vitesses d'échanges du cholestérol libre entre le plasma et le foie, les flux nets de cholestérol estérifié plasma-foie ou plasmacarcasse sont 10 fois moins rapides que les flux d'échanges du cholestérol libre entre ces milieux.

\section{RÉGULATION DE LA BIOSYNTHĖSE DES ACIDES BILIAIRES}

Les vitesses de sortie du cholestérol hors de son système (excrétion fécale du cholestérol et biosynthèse des acides biliaires) chez le rat, le porc et l'homme sont reportées dans le tableau I. La biosynthèse des acides biliaires exprimée en $\mathrm{mg} \cdot \mathrm{j}^{-1} \cdot \mathrm{kg}^{-1}$ est de 40 chez le rat, de 20 chez le porc

Fig 4. Elaboration d'un modèle du système cholestérol du rat. Celui-ci est établi en 5 étapes ( $T$ Magot, 1989). Les 4 premières (a, b, c, d) décrivent spécifiquement, grâce à des introductions variables de marqueur, une partie du modèle. a : absorption du cholestérol alimentaire et son déversement plasmatique; $b$ : échanges de cholestérol libre entre le plasma et les hématies; $c$ : mouvements de cholestérol estérifié a l'intérieur du plasma; $d$ : mouvements de cholestérol libre et estérifié entre le plasma et les différents organes. e : modèle complet obtenu par raccordement des 4 modèles précédents. CHL, CHE et CHT : cholestérol libre, estérifié et total. Les valeurs entre parenthèses représentent les paramètres des processus $\left(e \mathrm{~h}^{-1}\right.$ ), celles entre crochets les abondances de cholestérol (en $\mathrm{mg}$ ). 
Vitesses des mouvements de $\mathrm{CHL}\left(\mathrm{mg} \cdot \mathrm{h}^{-1}\right)$

(RAT)

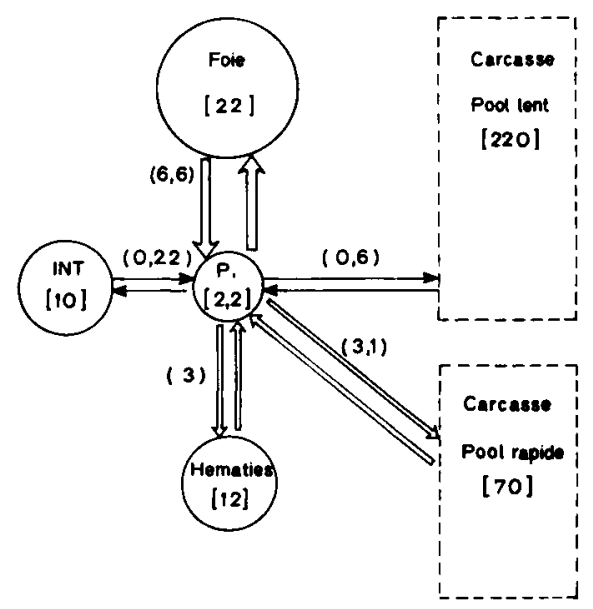

Fig 5. Modèle simplifié (de la figure 4) des mouvements de cholestérol libre chez le rat. Les valeurs entre crochets correspondent aux quantités (en $\mathrm{mg}$ ) de cholestérol libre dans les compartiments; les vitesses (en $\mathrm{mg}^{-h^{-1}}$ ) des mouvements de cholestérol libre entre les compartiments sont indiquées entre parenthèses.

mais n'atteint pas 10 chez l'homme. Elle est, en revanche, assez semblable pour ces 3 espèces quand on l'exprime pour $100 \mathrm{kcal}$ ingérées. L'excrétion fécale de cholestérol, $7 \mathrm{mg} . \mathrm{j}^{-1} \cdot \mathrm{kg}^{-1}$ chez le rat et l'homme, n'atteint que $3,3 \mathrm{mg} . \mathrm{j}^{-1} . \mathrm{kg}^{-1}$ chez le porc. Exprimée pour $100 \mathrm{kcal}$ ingérées, elle est inférieure à 4 chez le rat, atteint 6,7 chez le porc et 25 chez l'homme. Soulignons l'importance relative de la transformation du cholestérol en acides biliaires comme voie de sortie du cholestérol chez le rat et le porc qui rend compte de 80 à $85 \%$ de son élimination quotidienne. Chez l'homme, l'excrétion fécale du cholestérol et la biosynthèse des acides biliaires sont équivalentes.
Vitesses des mourements de CHE (mg. $\left.h^{-4}\right)$

(RAT)

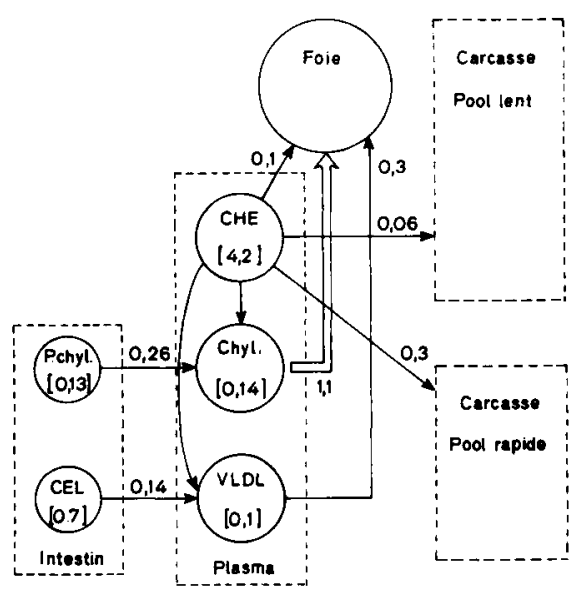

Fig 6. Modèle simplifié (de la figure 4) des mouvements de cholestérol estérifié chez le rat. Les valeurs entre crochets correspondent aux quantités (en mg) de cholestérol estérifié des compartiments; les vitesses (en $\mathrm{mg} \mathrm{h}^{-1}$ ) des mouvements de cholestérol estérifié entre les compartiments sont indiquées entre parenthèses.

Nous avons observé, à partir de plus de 60 expériences nutritionnelles diverses chez le rat, que la vitesse de transformation du cholestérol en acides biliaires est proportionnelle à la somme des entrées de cholestérol dans l'organisme (absorption plus sécrétion interne). Pour une très large gamme (5 à $100 \mathrm{mg.j}^{-1}$ ), la biosynthèse des acides biliaires répond aussi bien à une élévation du débit de cholestérol absorbé (à sécrétion interne constante) qu'à celle du débit de la synthèse (à absorption constante). Cela est la réponse dynamique d'un système ouvert non régulé (Mathé et Lutton, 1984). En d'autres termes, la biosynthèse des acides biliaires du rat nous paraît être un processus extrêmement sen- 
Tableau l. Débits moyens (en mg·j-1 $\mathrm{kg}^{-1}$ et en $\mathrm{mg}^{-1} \mathrm{j}^{-1}$ pour $100 \mathrm{kcal}$ ) de l'excrétion fécale du cholestérol et de sa transformation en acides biliaires chez le rat, le porc et l'homme.

\begin{tabular}{llll} 
& Rat & Porc & Homme \\
\hline $\begin{array}{l}\text { Excrétion fécale } \\
\left(\mathrm{mg}^{-1} \cdot \mathrm{kg}^{-1}\right)\end{array}$ & $7,1(16 \%)$ & $3,3(14 \%)$ & $7,1(50 \%)$ \\
$\begin{array}{l}\text { Transformation en acides biliaires } \\
\left(\mathrm{mg} \cdot \mathrm{j}^{-1} \cdot \mathrm{kg}^{-1}\right)\end{array}$ & $37,7(84 \%)$ & $20,7(86 \%)$ & $7,1(50 \%)$ \\
$\begin{array}{l}\text { Excrétion fécale } \\
\left(\mathrm{mg} \cdot{ }^{-1} \text { pour } 100 \mathrm{kcal}\right)\end{array}$ & 3,8 & 6,7 & 25 \\
$\begin{array}{l}\text { Transformation en acides biliaires } \\
\left(\mathrm{mg} \cdot \mathbf{j}^{-1} \text { pour } 100 \mathrm{kcal}\right)\end{array}$ & 20,3 & 41,2 & 25 \\
\hline
\end{tabular}

Entre parenthèses : pourcentage des sorties totales de cholestérol (excrétion fécale + transformation en acides blliaires).

sible qui s'adapte aux contingences du système cholestérol. A la suite des travaux de Mosbach, en particulier, une hypothèse qui a connu beaucoup de succès a été celle d'une régulation "feedback» de la biosynthèse hépatique des acides biliaires par leur propre flux retournant au foie. Fondée essentiellement sur le fait que la biosynthèse des acides biliaires est stimulée par la suppression de la circulation entérohépatique et rétro-inhibée par l'infusion d'acides biliaires abondants, cette notion est maintenant critiquée quand on considère des concentrations "physiologiques" d'acides biliaires, dans les expériences in vivo (Davis et al, 1988) ou même des cellules en culture (Botham et al, 1981). D'ailleurs, les travaux de Riottot et Sacquet (Riottot, 1987) portant sur plusieurs dizaines de conditions alimentaires différentes n'ont permis d'établir aucune corrélation entre la biosynthèse et la taille du pool des acides biliaires.
Si l'on infuse de la mévilonine (inhibiteur de la synthèse du cholestérol) avec du taurocholate, la synthèse des acides biliaires et la sécrétion de cholestérol dans la bile sont réduites (Davis et al, 1988). Certains auteurs ont alors pensé qu'un lien existait entre la HMG CoA réductase et la cholestérol-7 $\alpha$-monooxygénase dans le foie. Dans beaucoup de situations, en effet, la synthèse et la dégradation du cholestérol varient ensemble. II existe, cependant, un cas de découplage flagrant entre les deux processus : l'apport de cholestérol alimentaire provoque une inhibition de I'HMG CoA réductase hépatique tandis que la cholestérol- $7 \alpha$-monooxygénase est fortement stimulée. Dans un article récent, Björkhem et Akerlund (1988) constatent que ni la concentration du cholestérol des microsomes, ni le degré de saturation du substrat, ni le niveau de I'HMG CoA réductase ne paraissent être les régulateurs majeurs directs de l'activité cholestérol-7 $\alpha$ monooxygénase du foie du rat. 
L'apport de cholestérol exogène, qui induit une forte stimulation de la cholestérol$7 \alpha$-monooxygénase, se traduit par une augmentation importante de la production intestinale de chylomicrons et de VLDL rapidement captés par le foie. Plusieurs auteurs se sont récemment demandés si l'arrivée en masse de ces particules au foie stimulait la biosynthèse des acides biliaires. Dans des expériences de courte durée (1-2 h) sur le foie perfusé (Van Zuiden et al, 1987) ou entier (Stone et al, 1987), l'arrivée de lipoprotéines légères au foie est suivie d'une augmentation du stockage des esters de cholestérol, d'une élévation de l'activité $A C A T$, d'une sécrétion des VLDL par le foie, mais non d'une augmentation de la sécrétion de cholestérol dans la bile. Travaillant sur des cultures d'hépatocytes de rat, Mackinnon et al (1987) ont observé que la synthèse des acides biliaires est encore plus forte en présence de $H D L_{E}$ de rat qu'en présence de HDL de rat ou d'homme, pauvres en apo $E$. De plus, la méthylation qui empêche la liaison aux récepteurs impliquant l'apo $E$ supprime la stimulation. Enfin, ces HDL à apo $E$ inhibent l'incorporation d'eau tritiée dans les stérols de la cellule et provoquent une accumulation notable de cholestérol estérifié dans l'hépatocyte. Soulignons que le rat, espèce dont on vient de montrer l'extrême adaptabilité de la biosynthèse des acides biliaires, est également une espèce dont les lipoprotéines contiennent une forte proportion d'apo $\mathrm{E}$ tandis que le hamster ou l'homme, dont la biosynthèse des acides biliaires paraît moins modulable, possèdent beaucoup moins d'apo $E$ dans leurs lipoprotéines plasmatiques.

Il a été bien démontré que la sécrétion de cholestérol dans la bile est largement dépendante de celle des acides biliaires (Turley et Dietschy, 1979). Mais les travaux plus récents suggèrent que la capaci- té d'un acide biliaire à solubiliser et entraîner le cholestérol dans la bile est liée à son hydrophobicité (ou lipophilicité), comme l'on en juge par son comportement en HPLC (Bilhartz et Dietschy, 1988; Heuman et al, 1988). Les principaux acides biliaires de la bile du rat, du porc et de l'homme sont portés dans le tableau II. La bile du rat, dont la concentration en cholestérol est faible, contient des acides biliaires principaux peu hydrophobes entraînant mal le cholestérol. Il en est de même pour la bile du porc. Pour ces deux espèces, nous avons vu que le rapport excrétion fécale de cholestérol/biosynthèse des acides biliaires est faible ( $1 / 5$ à $1 / 4$, tableau I). Chez l'homme au contraire, la concentration du cholestérol biliaire est plus élevée, les acides biliaires de la bile sont en moyenne plus hydrophobes. Dans cette espèce, le débit d'excrétion fécale de cholestérol atteint celui de la biosynthèse des acides biliaires (tableau I).

\section{RÉGULATION \\ DE LA CHOLESTÉROGENÈSE ET DE LA CHOLESTÉROLÉMIE}

Exprimée en $\mathrm{mg} / \mathrm{j}$ par $\mathrm{kg}$ de poids corporel, la synthèse de cholestérol atteint plus de 40 chez le rat, est deux fois moins abondante chez le porc et 5-6 fois moindre chez l'homme, mais calculée pour $100 \mathrm{kcal}$ ingérées, les valeurs de la synthèse deviennent proches pour ces 3 espèces (tableau III). Deux organes, le foie et l'intestin grêle, sont les principaux responsables de la synthèse de cholestérol, déversé dans le système cholestérol du rat (sécrétion dite interne, Lutton, 1976). L'addition de $0,5 \%$ de cholestérol exogène provoque une faible inhibition (20-30\%) de la cholestérogenèse intestinale, mais réduit pratiquement à néant la synthèse de cholestérol dans le foie (Perrodin et Lutton, 1985). 
Tableau II. Rapport molaire acides biliaires/cholestérol, composition (en \%) et index d'hydrophobicité des acides biliaires principaux, de la bile chez le rat, le porc et l'homme.

\begin{tabular}{|c|c|c|c|c|}
\hline & & Bile & & Hydrophobilicté \\
\hline & $\begin{array}{l}\text { Rapport } \\
\text { molaire } \\
\mathrm{AB} / \mathrm{CH}\end{array}$ & $\begin{array}{l}\text { Acides biliaires } \\
\text { principaux }\end{array}$ & $\%$ & \\
\hline Rat & $50-100$ & $\begin{array}{l}\beta \text {-muricholique } \\
\text { Cholique } \\
\text { Ursodésoxycholique } \\
\text { Chénodésoxycholique }\end{array}$ & $\begin{array}{c}50-55 \\
35-40 \\
4 \\
4\end{array}$ & $\begin{array}{r}-0,40 \\
0,23 \\
-0,17 \\
0,83\end{array}$ \\
\hline Porc & $130-160$ & $\begin{array}{l}\text { Hyocholique } \\
\text { Hyodésoxycholique } \\
\text { Chénodésoxycholique }\end{array}$ & $\begin{array}{l}35-40 \\
25-30 \\
30-35\end{array}$ & $\begin{array}{r}-0,03 \\
0,09 \\
0,83\end{array}$ \\
\hline Homme & 25 & $\begin{array}{l}\text { Cholique } \\
\text { Chénodésoxycholique } \\
\text { Désoxycholique } \\
\text { Lithocholique }\end{array}$ & $\begin{array}{l}35-40 \\
35-40 \\
20-25 \\
1-2\end{array}$ & $\begin{array}{l}0,23 \\
0,83 \\
0,98 \\
1,73\end{array}$ \\
\hline
\end{tabular}

L'inhibition de la cholestérogenèse hépatique est la conséquence de l'arrivée massive au foie des "remnants» de chylomicrons et de VLDL intestinales contenant de l'apo $B_{\mid}$(ou apo $B_{48}$ ) synthétisée exclusivement ou principalement par l'intestin, selon les espèces (Nervi et al, 1975; Lusis, 1988). Le travail de modélisation du système cholestérol du rat entrepris précédemment dans le laboratoire (Magot, 1989) a mis en évidence les rôles antago- nistes de l'intestin et du foie dans le métabolisme du cholestérol estérifié chez cet animal (fig 4). L'importante contribution de l'intestin à la formation du cholestérol estérifié des VLDL chez le rat est à mettre en parallèle avec l'importante contribution de cet organe à la synthèse du cholestérol. Le marquage du système à travers l'intestin par l'intermédiaire du cholestérol alimentaire ou du cholestérol de synthèse intestinale provoque peu de marquage des LDL,

Tableau III. Débits moyens (en $\mathrm{mg} \cdot \mathrm{j}^{-1} \cdot \mathrm{kg}^{-1}$ et en $\mathrm{mg}^{-1}{ }^{-1}$ pour $100 \mathrm{kcal}$ ) de synthèse du cholestérol chez le rat, le porc et l'homme.

Rat Porc Homme

Synthèse de cholestérol

$\left(\mathrm{mg} \cdot \mathrm{j}^{-1} \cdot \mathrm{kg}^{-1}\right)$

$\left(\mathrm{mg} \cdot \mathrm{j}^{-1} \cdot\right.$ pour $\left.100 \mathrm{kcal}\right)$

$\begin{array}{llc}41,4 & 23,4 & 7,1 \\ 22,3 & 46,6 & 25\end{array}$


puisque les VLDL intestinales du rat (riches en apo $B_{48}$ ) sont très rapidement captées par le foie. Les VLDL d'origine hépatique, en revanche, qui contiennent plus d'apo $B_{h}$ (ou apo $B_{100}$ ), ont une durée de vie plus longue dans le plasma (tableaux IV et V) et peuvent conduire par transformation intraplasmatique aux IDL puis aux LDL à renouvellement plus lent. C'est à cette faible seconde voie, $10 \%$ de la précédente chez le rat en période d'absorption selon Magot (1989), que l'on attribue le fait que les LDL sont si faiblement représentées chez cet animal. Chez l'homme, au contraire, il existe une faible concentration d'apo $\mathrm{B}_{\mathbf{4 8}}$ dans le plasma et les VLDL plasmatiques seront en grande partie transformées en LDL (Eisenberg et al, 1973; Sigurdsson et al, 1975), d'où l'importance de cette dernière classe de lipoprotéines dans le plasma humain.

Cette description qui oppose lipoprotéines légères d'origine hépatique et d'origine intestinale nous semble un facteur important de la régulation de la concentration du cholestérol plasmatique. En effet, des travaux précédents du laboratoire ont mis en évidence une relation inverse entre la cholestérolémie et la sécrétion interne de cholestérol quand la synthèse intestinale est importante (Chevallier et al, 1976; Lutton, 1980); au contraire, la stimulation de la synthèse du cholestérol dans le foie s'accompagne d'une élévation de la cholestérolémie (Mathé et Lutton, 1984; Sérougne et al, 1987). Dans le cas où la synthèse intestinale de cholestérol est importante, nous avons aussi observé une relation inverse entre sécrétion interne et coefficient d'absorption du cholestérol alimentaire. Or, Davidson et al (1987) ont montré une corrélation identique entre la synthese intestinale d'apo $B_{48}$ et le coefficient d'absorption du cholestérol du lumen, mais non avec l'entrée de triglycérides. Ainsi, une réduction du flux de cholestérol exogène dans l'entérocyte pourrait-elle provoquer une augmentation de la synthèse du cholestérol et de l'apo $B_{48}$ dans l'entérocyte, une stimulation de la sécrétion des VLDL intestinales captées rapidement par le foie et donnant naissance à moins de lipoprotéines plasmatiques de densité plus élevée $\left(\mathrm{LDL}_{2}, \mathrm{HDL}_{1}, \ldots\right)$, aboutissant à une baisse de la cholestérolémie. Dans certaines hypercholestérolémies et hyperlipoprotéinémies, au contraire, la synthèse hépatique de cholestérol est très stimulée : c'est le cas de l'hypercholestérolémie génétique du rat RICO

Tableau IV. Taux de catabolisme $\left(\right.$ pool ${ }^{-1}$ ) du cholestérol ester des lipoprotéines plasmatiques chez le rat et l'homme.

\begin{tabular}{lll}
\hline & Rat & Homme \\
\hline Chylo-CE & $7-10 \mathrm{a}$ & \\
VLDL-CE (lymphe) & $4,5-4,9 \mathrm{a}$ & \\
(plasma) & $3,4-3,6 \mathrm{a}$ & $0,3-0,6 \mathrm{~b}, \mathrm{c}$ \\
LDL-CE & $0,06-0,15$ & $0,02-0,04 \mathrm{~b}, \mathrm{c}$ \\
HDL-CE & $0,1-0,3 \mathrm{a}, \mathrm{a}$ & $0,1-0,13^{\mathrm{b}, \mathrm{d}}$ \\
& & \\
\hline
\end{tabular}

a : K Ouguerram, T Magot, C Lutton (non publié); b : T Magot et al (soumis); c : Monroe et al (1983); d : Schwartz et al (1982); e : Van't Hooft et al (1981). 
Tableau V. Taux de catabolisme $\left(\right.$ pool $\left.h^{-1}\right)$ des apolipoprotéines des lipoprotéines plasmatiques chez le rat et l'homme.

Rat Homme

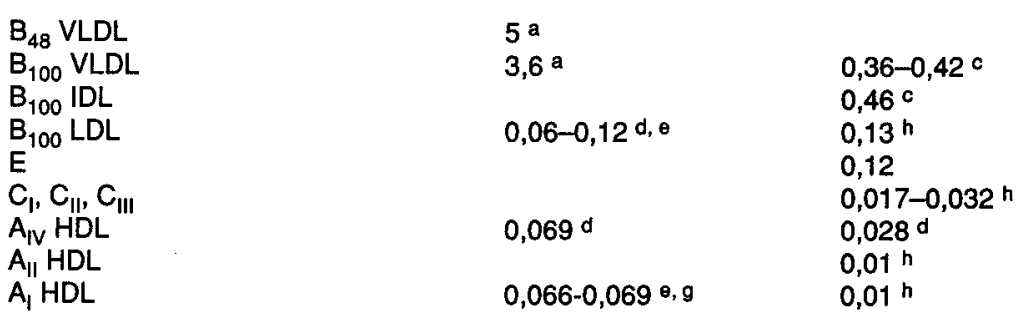

a : Elovson J et al (1982); $b$ : Huff MW, Telford (1985); c : Thompson et al (1984); d : Lefevre, Roheim (1984); $\theta$ : Ouguerram, Magot, Lutton (non publié); $f$ : Carew et al (1982); $g$ : Fidge (1980); h: Malmendier et al (1986 et 1988 ).

ou de l'hypercholestérolémie alimentaire déclenchée par l'addition de cystine (Cardona-Sanclemente et al, 1988; Sérougne et al, 1988), où l'on observe dans les lipoprotéines légères une augmentation concomitante des proportions des apolipoprotéines synthétisées surtout dans le foie (apo $B_{100}$, apo $E, \ldots$ ) au détriment de l'apo $\mathrm{B}_{48}$ d'origine intestinale.

Ainsi, l'importance relative de la synthèse du cholestérol par le foie et l'intestin sur la modulation de la cholestérolémie importance que nous avions soulignée chez le rat - est probablement liée à leur plus ou moins grande aptitude à sécréter des apolipoprotéines à renouvellement rapide (intestin) ou lent (foie). Ces observations ont sans doute une portée générale, car les espèces qui répondent à un accroissement du cholestérol alimentaire par une forte élévation de leur cholestérolémie (cobaye, lapin) sont également celles chez qui la synthèse intestinale du cholestérol est efficacement inhibée. II n'en est pas de même pour les espèces répondant «mal» au cholestérol exogène (rat, chien, ...).
Dans l'espèce humaine également existent des sujets hyper et hypo répondeurs au cholestérol exogène ou aux acides gras saturés qui présentent des réponses très reproductibles pour chaque sujet (Katan et al, 1988). Chez un sujet hypercholestérolémique de type Ila, l'activité de I'HMG CoA réductase intestinale était moitié moindre que celle du normocholestérolémique (Freeman et al, 1988).

\section{REMERCIEMENTS}

L'auteur tient à remercier plus particulièrement ses principaux collaborateurs impliqués dans les études de la biodynamique du cholestérol, la régulation de la cholestérolémie, la modélisation du système cholestérol (Mesdames J Férézou, C Sérougne, Messieurs T Magot, G Champarnaud) ou la biodynamique des acides biliaires (Messieurs M Parquet et M Riottot), ainsi que les jeunes chercheurs qui ont participé activement à la vie scientifique du laboratoire au cours des 3 dernières années (T Hajri, J Khallou, V Legrand-Defretin, $K$ Ouguerram). La précieuse assistance de Madame C Verneau pour le dépouillement chimique des expériences, de Ma- 
dame $L$ Boivin pour la frappe du manuscrit et de Monsieur Moqué pour la réalisation des illustrations est vivement appréciée.

Ces travaux ont fait l'objet du concours financier de la Caisse régionale d'assurance-maladie de l'lle de France (CRE INSERM du 25 janvier 1989).

\section{RÉFÉRENCES}

Bergner PT (1964) Tracer dynamic and the determination of pool size and turnover factors in metabolic systems. J Theor Biol 6, 137158

Bilhartz LE, Dietschy JM (1988) Bile salt hydrophobicity influences cholesterol recruitement from rat liver in vivo when cholesterol synthesis and lipoprotein uptake are constant. Gastroenterology 95, 771-9

Björkhem IB, Akerlund JE (1988) Studies on the link between HMG-COA reductase and cholesterol-7 $\alpha-\mathrm{OH}$-ase in rat liver. $J$ Lipid Res 29, 136-141

Botham KM, Lawson ME, Beckett GJ, PercyRobb IW, Boyd GS (1981) The effect of portal blood concentration on bile salt synthesis in rat liver. Biochem Biophys Acta 666, 237245

Cardona-Sanclemente LE, Férézou J, Lutton C (1988) Cholesterol metabolism in the genetically hypercholesterolemic rat (RICO). II. Study of plasma lipoproteins and effect of dietary cholesterol. Biochem Biophys Acta 960, 382-389

Carew TE, Beltz WF (1982) Kinetics of low density lipoproteins labeled with $\left({ }^{14} \mathrm{C}\right)$ sucrose: theoretical basis of a method for tracing the sites of apoprotein degradation in vivo. In: Lipoprotein kinetics and modeling (M Berman, SM Grundy, BV Howard, eds), Acad Press, New York, 169-179

Champarnaud G, Magot T, Lutton C (1987) A microcomputer program for simulation of linear compartmental physiological models: computation on Apple lle. Comput Methods Program Biomed 24, 63-64

Chevallier F (1956) L'espace cholestérol du rat. Arch Sci Physiol 10, 249-275

Chevallier F (1967a) Le cholestérol : Notions de base et problèmes. Cah Nutr Diét 2, 5-20
Chevallier F (1967b) Dynamics of cholesterol in rats, studied by the isolopic equilibrium method. Adv Lipid Res 5, 209-239

Chevallier F, Lutton C (1973) The intestine is the major site of cholesterol synthesis in the rat. Nat New Biol 242, 61-62

Chevallier F, Magot T (1975) Evidence of major role of the intestine in cholesterol synthesis in the adult male rat. Experientia 31, 627-629

Chevallier F, Mathé D, Lutton C (1976) Lois des taux de cholestérol dans le plasma et le foie de rats adultes. CR Séances Acad Sci sér $D$ 283, 1759-1762

Davidson NO, Magun AM, Brasitus TA, Glickman RM (1987) Intestinal apolipoprotéin $A_{1}$ and $\mathrm{B}_{48}$ metabolism: effects of sustained alterations in dietary triglyceride and mucosal cholesterol flux. J Lipid Res 28, 388-402

Davis RA, Musso CA, Malone-McNeal M, Lattier GR, Hyde PM, Archambault-Schexnayder J, Straka M (1988) Examination of bile acid negative feedback regulation in rats. $J$ Lipid Res 29, 202-211

Duane WC, McHale AP, Hamilton JN (1988) Studies of feedback suppression of bile salt synthesis in the bile fistula rat. $J$ Lipid Res 29, 212-214

Eisenberg S, Bilheimer DW, Levy LI (1973) On the metabolic conversion of plasma very low density lipoproteins to low density lipoproteins. Biochem Biophys Acta 326, 361-377

Elovson J, Baker N, Kannan R, Dokhtens M (1982) Molecular and kinetic nonidentity of two apoprotein B peptide classes in rat plasma VLDL, IDL and LDL. In: Lipoprotein kinetics and modeling (M Berman, SM Grundy, BV Howard, eds) Acad Press, New York, 145-156

Elovson J, Huang YO, Baker N, Kannan R (1981) Apolipoprotein B is structurally and metabolically heterogeneous in the rat. Proc Natl Acad Sci USA 78, 157-161

Férézou J, Huc D, Rautureau J, Coste T, Lutton C (1987) Origine du cholestérol biliaire chez l'homme. Gastroenterol Clin Biol 11, 811-812

Férézou J, Coste T, Huc D, Rautureau J, Lutton C (1989) Origine du cholestérol biliaire chez l'homme : effet du drainage biliaire après cholecystectomie. Gastroenterol Clin Biol (sous presse) 
Fidge NH (1980) The redistribution and metabolism of iodinated apolipoprotein $A_{I V}$ in rats. Biochem Biophys Acta 619, 129-141

Freeman ML, Prigge WF, Hunninghake DB, Duane WC, Gebhard RL (1988) Intestinal HMG CoA reductase activity is low in hypercholesterolemic patients and is further decreased with lovastatin therapy. J Lipid Res 29, 839845

Heuman DM, Vhlacevic ZR, Bailey ML, Hylemon PB (1988) Regulation of bile acid synthesis. II. Effect of bile acid feeding on enzymes regulating hepatic cholesterol and bile acid synthesis in the rat. Hepatology 8, 892897

Huff MW, Telford DE (1985) Direct synthesis of low-density lipoprotein apoprotein $B$ in the miniature pig. Metabolism Clin Exp 34, 36-42

Katan MB, Berns MAM, Glatz JFC, Knuiman JT, Nobels A, De Vries JHM (1988) Congruence of individual responsiveness to dietary cholesterol and to saturated fat in humans. $J$ Lipid Res 29, 883-892

Lefevre M, Roheim PS (1984) Metabolism of apolipoprotein $A_{I V}$. $J$ Lipid Res 25, 16031610

Lusis AJ (1988) Genetic factors affecting blood lipoproteins: the candidate gene approach. $J$ Lipid Res 29, 397-429

Lutton C (1976) The role of digestive tract in cholesterol metabolism. Digestion 14, 342356

Lutton C (1980) New aspects of cholesterol homeostasis. In: Advances in Experimental Medicine: a centenary tribute to Claude Bernard (H Parvez, S Parvez, eds) Elsevier, Amsterdam, 499-526

Lutton C, Chevallier F (1972) Vitesses des processus de renouvellement du cholestérol contenu dans son espace de transfert, chez le rat. III. Modifications et étude critique de la méthode d'équilibre isotopique. Biochim Biophys Acta 255, 762-779

Mackinnon AM, Drevon CA, Sand TM, Davis RA (1987) Regulation of bile acid synthesis in cultured rat hepatocytes: stimulation by apo E-rich HDL. J Lipid Res 28, 847-855

Magot T (1985) Origine et devenir du cholestérol des lipoprotéines plasmatiques du rat in vivo. III. Mise en évidence et mesure du flux de cholestérol estérifié des HDL vers les chylomicrons. Ann Nutr Metab 29, 175-183

Magot T (1989) A multicompartmental model of cholesterol metabolism in rats. J Physiol Paris 83, 80-94

Magot T, Chevallier F (1979) Measurement of the rate of cholesterol synthesis in various organs of the rat in vivo. Ann Biol Anim Biochim Biophys 19, 1757-1770

Magot T, Verneau C, Lutton C, Chevalier $F$ (1985) Origin and fate of cholesterol in rat plama lipoproteins in vivo. I. Qualitative analysis. Ann Nutr Metab 29, 147-159

Magot T, Malmendier CL, Ouguerram K, Lontie JF, Lutton C. In vivo effect of simvastatin on lipoprotein cholesteryl ester metabolism in normocholesterolemic subjects. Clin Chim Acta (soumis pour publication Clin Chim Acta)

Malmendier CL, Lontie JF, Grutman G, Delcroix C (1986) Metabolism of apolipoprotein $C_{1}$ in normolipoproteinemic human. Atherosclerosis 62, 167-172

Malmendier CL, Lontie JF, Grutman G, Delcroix C (1986) Metabolism of apolipoprotein $C_{111}$ in normolipoproteinemic human. Atherosclerosis 69, 51-59

Mathé D, Lutton C (1984) Le cholestérol. Aspects dynamiques et métaboliques. J Physiol (Paris) 79, 41-97

Monroe $P$, Vlahcevic $Z R$, Swell L (1983) In vivo evaluation of lipoprotein cholesterol ester metabolism in patients with liver disease. Gastroenterology 85, 820-829

Nervi FO, Weis HJ, Dietschy JM (1975) The kinetic characteristics of inhibition of hepatic cholesterogenesis by lipoproteins of intestinal origin. J Biol Chem 250, 4145-4151

Perrodin M, Lutton C (1985) In vivo cholesterol synthesis by the rat digestive tract. I. A topological study. Reprod Nutr Dev 25, 647-657

Riottot M (1987) Métabolisme des acides biliaires chez le rat. Influence de la flore microbienne du tractus digestif et des glucides alimentaires. Thèse de Doctorat d'Etat, Université Paris Sud, Orsay, $n^{\circ} 3411$

Schwartz CC, Vlahcevic ZR, Berman M, Meadows JG, Nisman RM, Swell L (1982) Central role of high density lipoprotein in plasma free cholesterol metabolism. J Clin invest 70, 105-116 
Sérougne C, Férézou J, Rukaj A (1987) A new relationship between cholesterolemia and cholesterol synthesis determined in rats fed an excess of cystine. Biochim Biophys Acta $921,522-530$

Sérougne C, Mathé D, Lutton C (1988) Induction of long-lasting hyper-cholesterolemia in the rat fed a cystine-enriched diet. Lipids 23 , 930-936

Sigurdsson G, Nicoll A, Lewis B (1975) Conversion of very low density lipoprotein to low density lipoprotein. J Clin Invest 56, 1481 1490

Stone BG, Schreiber D, Alleman LD, Ho CY (1987) Hepatic metabolism and secretion of a cholesterol enriched lipoprotein fraction. $J$ Lipid Res 28, 162-172

Thompson GR, Myant NB, Soutar AK (1984) Turnover of VLDL and IDL-Apo $B$ in controls and patients with familial hypercholesterolemia. In : Latent Dyslipoproteinemias and atherosclerosis (De Gennes et al, eds), Raven Press, New York, 87-92

Turley SD, Dietschy JM (1979) Regulation of biliary cholesterol output in the rat: dissociation from the rate of hepatic cholesterol synthesis, the size of the hepatic cholesteryl ester pool, and the hepatic uptake of chylomicrons cholesterol. J Lipid Res 20, 923-934

Van'T Hooft FM, Van Gent T, Van Tol A (1981) Turnover and uptake by organs of radioactive serum high-density lipoprotein cholesteryl esters and phospholipids in the rat in vivo. Biochem J 196, 877-885

Van Zuiden PEA, Cooper AD, Erikson SK (1987) Regulation of rat hepatic cholesterol metabolism. Effects of lipoprotein composition in vivo and in the perfused liver and on hepatic secretion. J Lipid Res 28, 930-940 